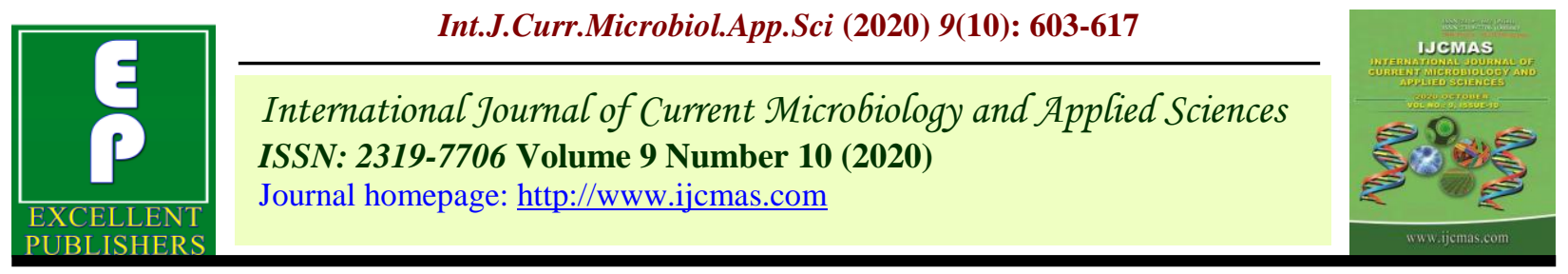

Original Research Article

https://doi.org/10.20546/ijcmas.2020.910.072

\title{
Morphological Characterization and Diversity Analysis of Adzukibean [Vigna angularis (Willd.) Ohwi and Ohashi] Germplasm
}

\author{
Anjali* and Neelam Bhardwaj \\ Department of Genetics and Plant Breeding, CSK Himachal Pradesh KrishiVishvavidyalaya, \\ Palampur-176062 (H.P.) India \\ *Corresponding author
}

\section{A B S T R A C T}

Keywords

characterization, Clustering, Adzuki bean, Diversity

Article Info

Accepted:

07 September 2020

Available Online:

10 October 2020
One hundred and fifty-one genotypes of adzuki bean were evaluated at the experimental farm of the Department of Genetics and Plant Breeding, CSKHPKV, Palampur in Augmented Block Design during Kharif, 2019 for characterization and diversity analysis using 22 agro-morphological characters. 5 genotypes viz., EC-340286, EC-340267, EC-340271, EC000263 and EC-340251 showed resistance to Cercospora leaf spot disease. Cluster analysis grouped these genotypes into ten clusters with cluster III having highest 36 genotypes. Sufficient variability was observed in the genotypes studied based on characterization and cluster analysis which could be utilized in breeding programmes of this crop.

\section{Introduction}

Adzuki bean [Vigna angularis (Willd.) Ohwi and Ohashi] is a self-pollinated legume crop $(2 \mathrm{n}=22)$ belonging to the genus Vigna of thefamily Leguminosae. Its center of origin is East Asia andit is mainly cultivated in China, Japan, Taiwan, South Korea, Bhutan, India, Nepal, US, South America, New Zealand and Africa. $V$. angularis var. nipponensis is the presumed wild ancestor of cultivated adzuki bean (Yamaguchi et al., 1992). This wild species is distributed across a wide area of Japan, the Korean and China peninsula, including Taiwan, Nepal and Bhutan
(Vaughan et al., 2004). China is the largest producer of adzuki bean in the world with an estimated production of about 260 thousand tons in the year 2019-20. In Japan, it is the second most important legume after soybean with a consumption of about 140,000 tonnes per year (Anonymous, 2019). Outside East Asia, Canada and Australia have also started producing adzuki bean in recent years, mainly for export reasons. In India, it is considered as an underutilized crop and its cultivation is limited to the North-eastern and Northern hill zones. It occasionally grows in Chamba, Kangra, Mandi and Bilaspur districts of Himachal Pradesh where it is mainly grown 
as an intercrop with maize (Shweta, 2013). It is an annual vine with trifoliate leaves, yellow flowers, slender and curved pods, determinate and late maturing. Its seed coat colour is generally wine red but black, white, yellow or mottled colour also exists. It is consumed in the form of whole seed, split grain and flour while, immature pods and leaves are eaten as vegetable (Dua et al., 2009). In India, it is taken as a pulse crop but in Japan and China it is widely used in a variety of foods like cake, porridge, desserts, adzuki rice, jelly, adzuki milk and ice cream. It is an important legume crop of temperate and sub temperate regions grown for human consumption due to its high nutritional values and broad adaptability. It improves soil condition by nitrogen fixation up to the tune of $100 \mathrm{~kg} / \mathrm{ha}$. It is thus evident that adzuki bean has the potential of being an agriculturally important crop and has large scope in future to meet the demand of pulses.

The low genetic variability observed in the crop demands the strengthening of breeding programmes through introduction of new germplasm, collection of local genotypes and adopting interspecific hybridization. So, there is an urgent need to characterize and evaluate adzuki bean genotypes to identify donor(s) for different traits and utilizing these genotypes in different breeding programmes. Germplasm plays the key role in providing needed attributes for developing superior varieties. Characterization involves estimating existing variability across the population of individuals (Franco and Hidalgo, 2003). Describing the characteristics of a crop species based on standard descriptors is helpful for better utilization and conservation of germplasm (Kumari et al., 2017). Moreover, morphological characterization studies play an important role in better understanding of crop diversity. Thus, it is essential to conserve the genotypes and explore the gene pool of adzuki bean for breeding purposes. Diversity analysis is an essential process for identification of the genetic relatedness of the available genetic resources and to display the trends associated with latitude of germplasm origin, which were positive or negative according to the trait (Wang et al., 2001). Mahalanobis $\mathrm{D}^{2}$-analysis (1936) is a very useful tool in studying the nature and magnitude of diversity present in the available germplasm. It helps in the selection of diverse parental combinations to produce segregating progenies with maximum genetic variability (Barrett and Kidwell, 1998) and introgressing desirable traits from diverse germplasm to cultivated varieties to broaden their genetic base (Thompson et al., 1998). Therefore, present investigation was undertaken for morphological characterization studies and to identify parents for future crop breeding programme for the improvement of this underutilized pulse.

\section{Materials and Methods}

The experimental material for the present study comprised of 151 adzuki bean genotypes along with 2 checks viz., Local Totru and HPU-51, evaluated at the experimental farm of Department of Genetics and Plant Breeding, CSKHPKV, Palampur during Kharif, 2019 for 22 agromorphological characters including 10 quantitative and 12 qualitative traits in Augmented Block Design (Federer, 1956) during Kharif, 2019 (Table 1). Each entry was raised in one rowed plot of $3 \mathrm{~m}$ length with row to row and plant to plant spacing of $30 \mathrm{~cm}$ and $10 \mathrm{~cm}$, respectively in 15 blocks. Each block consisted of 12 genotypes including 2 checks and the checks were allotted randomly in each block. The crop was raised following standard package of practices. Data was recorded on five randomly choosen plants for the traits viz., plant height $(\mathrm{cm})$, primary branches per plant, clusters per plant, pods per cluster, pods per plant, seeds per pod, 1000seed weight $(\mathrm{g})$ and seed yield per plant $(\mathrm{g})$ 
while, for days to $50 \%$ flowering and days to $80 \%$ maturity data was recorded on plot basis. Data was also recorded for the qualitative traits viz., early plant vigour, plant habit, leaf colour, leaf surface, leaflet shape, flower colour, stem colour, stem surface, pod angle, pod surface, seed coat colour and biotic stress susceptibility. Grouping of genotypes according to resistance against Cercospora leaf spot disease was done using 0-9 scale as per Alice and Nadarajan (2007).Genetic diversity studies were conducted as per Mahalanobis $\mathrm{D}^{2}$-analysis (1936).Frequency distribution of these traits was performed using StatistiXL version 1.10 software to determine the best relationships among characters and genotypes.

\section{Results and Discussion}

\section{Frequency distribution of morphological traits}

Qualitative traits were studied on the basis of visual observations and their frequency distribution revealed the presence of significant variation in adzuki bean germplasm [Fig. 1(a)-1(1)]. $77.8 \%$ of the total accessions had good nature of growth followed by $15.7 \%$ having very good plant vigour for the character early plant vigour [Fig. 1(a)]. All accessions i.e. 100\% showed indeterminate type of growth habit [Fig. 1(b)]. Three types of leaf colour were observed in the germplasm i.e. yellowish green, green and dark green. $52.3 \%$ plants were having green coloured leaves followed by $38.6 \%$ which were having yellowish green leaves [Fig. 1(c)]. Most of the accessions, $69.3 \%$ were having pubescent type of leaf surfaces [Fig. 1(d)]. About $63.4 \%$ accessions had entire shape of leaflet, while $34 \%$ were having lobed shaped leaflet [Fig. 1(e)]. For stem pigmentation, $94.8 \%$ of accessions were found to have green coloured stem [Fig. 1(f)]. $90.2 \%$ genotypes were having pubescent type of stem surface whereas, only $9.8 \%$ showed glabrous type of stem surface [Fig. 1(g)]. Regarding flower colour92.8\% accessions were having yellow coloured flowers while, $7.2 \%$ were having light yellow coloured flowers [Fig. 1(h)]. All accessions i.e. 100\% were having erect type of pod angle [Fig. 1(i)]. Maximum number of accessions i.e. 96.1\% had glabrous pod surface [Fig. 1(j)]. Seeds of adzuki bean germplasm have shown wide variation with respect to seed coat colour. Maximum accessions i.e. $80.4 \%$ possessed red seed coat colour, followed by $17.6 \%$ which showed other types of seed colour and $2 \%$ were having maroon type of seed coat colours [Fig. 1(k)]. $60 \%$ of the accessions were susceptible to the disease while, $31.4 \%$ had moderate susceptibility. Only $2 \%$ accessions showed resistance to the disease whereas, $2.6 \%$ accessions were moderately resistant to the disease.[Fig. 1(1)].

\section{Disease reaction}

Response to Cercospora leaf spot disease was observed among the accessions under open field condition (Table 2). Results revealed that 5 genotypes viz., EC-340286, EC340267, EC-340271, EC-000263 and EC340251 showed resistance to the disease. 9 genotypes viz., EC-000377, EC-340284, IC341960, EC-57959, IC-485385, EC-340277, IC-341951, IC-34193 and IC-341963 were found moderately resistant, 50 genotypes were found moderately susceptible, 85 were found susceptible and 4 genotypes were showing very high susceptibility to the disease.

Resistant genotypes can be used as donor parents for future breeding programme. Kaushal and Singh (1988) introgress the resistance of adzuki bean against fungal and viral diseases into urdbean by crossing them to achieve an interspecific hybrid using different non-conventional breeding methods. 
Table.1 List of germplasm lines of adzuki bean evaluated under the present study

\begin{tabular}{|c|c|c|c|c|c|c|c|}
\hline S. No. & Genotypes & \multicolumn{2}{|c|}{ S. No. Genotypes } & \multicolumn{2}{|c|}{ S. No. Genotypes } & \multicolumn{2}{|c|}{ S. No. Genotypes } \\
\hline 1 & EC-290251 & 41 & EC-24102 & 81 & EC-000271 & 115 & EC-34264 \\
\hline 2 & EC-187896 & 42 & EC-341943 & 82 & EC-340277 & 116 & EC-36070 \\
\hline 3 & IC-341948 & 43 & EC-340276 & 83 & IC-469174 & 117 & EC-18959 \\
\hline 4 & EC-340272 & 44 & EC-340280 & 84 & IC-341944 & 118 & IC-46917 \\
\hline 5 & EC-340258 & 45 & EC-59459 & 85 & EC-120480 & 119 & EC-240251 \\
\hline 6 & EC-15648 & 46 & EC-340279 & 86 & IC-241041 & 120 & EC-281186 \\
\hline 7 & EC-340286 & 47 & EC-340259 & 87 & EC-120466 & 121 & IC-485396 \\
\hline 8 & EC-120460 & 48 & EC-30250 & 88 & IC-469172 & 122 & EC-36973 \\
\hline 9 & IC-341957 & 49 & EC-340267 & 89 & EC-87896 & 123 & IC-30270 \\
\hline 10 & EC-340247 & 50 & IC-485886 & 90 & EC-340246 & 124 & IC-341940 \\
\hline 11 & IC-341951 & 51 & EC-187898 & 91 & IC-341956 & 125 & IC-24105 \\
\hline 12 & EC-340274 & 52 & EC-340268 & 92 & EC-341955 & 126 & IC-24522 \\
\hline 13 & EC-59489 & 53 & EC-000262 & 93 & IC-341952 & 127 & EC-000263 \\
\hline 14 & EC-340281 & 54 & EC-340249 & 94 & EC-340287 & 128 & EC-57959 \\
\hline 15 & EC-240246 & 55 & EC-34027 & 95 & IC-341939 & 129 & EC-340269 \\
\hline 16 & IC-251353 & 56 & EC-340283 & 96 & IC-341947 & 130 & IC-341942 \\
\hline 17 & EC-340275 & 57 & EC-340248 & 97 & EC-340278 & 131 & IC-0293 \\
\hline 18 & IC-341950 & 58 & EC-87071 & 98 & EC-340251 & 132 & IC-140846 \\
\hline 19 & IC-341953 & 59 & EC-340270 & 99 & EC-340244 & 133 & IC-34196 \\
\hline 20 & EC-340245 & 60 & IC-469175 & 100 & IC-89957 & 134 & IC-140845 \\
\hline 21 & IC-341949 & 61 & EC-340264 & 101 & IC-108855 & 135 & EC-34625 \\
\hline 22 & EC-340257 & 62 & IC-485385 & 102 & EC-340256 & 136 & EC-18151 \\
\hline 23 & EC-340265 & 63 & EC-340252 & 103 & IC-341954 & 137 & EC-341962 \\
\hline 24 & EC-000248 & 64 & EC-000276 & 104 & IC-341938 & 138 & EC-340266 \\
\hline 25 & IC-108856 & 65 & EC-15256 & 105 & IC-341937 & 139 & IC-341959 \\
\hline 26 & EC-000254 & 66 & EC-340250 & 106 & EC-87815 & 140 & IC-341960 \\
\hline 27 & EC-340240 & 67 & EC-340263 & 107 & EC-87895 & 141 & EC-340260 \\
\hline 28 & EC-57159 & 68 & EC-30253 & 108 & IC-140848 & 142 & EC-340262 \\
\hline 29 & EC-340254 & 69 & EC-30270 & 109 & IC-485382 & 143 & EC-340273 \\
\hline 30 & EC-000249 & 70 & EC-340261 & 110 & IC-485388 & 144 & EC-340255 \\
\hline 31 & EC-290652 & 71 & EC-340288 & 111 & EC-18256 & 145 & IC-108857 \\
\hline 32 & IC-341958 & 72 & EC-108080 & 112 & IC-341941 & 146 & EC-57459 \\
\hline 33 & IC-108854 & 73 & EC-24523 & 113 & IC-341946 & 147 & IC-455396 \\
\hline 34 & EC-340285 & 74 & EC-08707 & 114 & EC-000372 & 148 & EC-340271 \\
\hline 35 & EC-000251 & 75 & IC-16761 & 109 & IC-485382 & 149 & EC-80850 \\
\hline 36 & EC-340284 & 76 & IC-341945 & 110 & IC-485388 & 150 & EC-000264 \\
\hline 37 & EC-340282 & 77 & IC-469173 & 111 & EC-18256 & 151 & EC-15257 \\
\hline 38 & EC-340253 & 78 & IC-341963 & 112 & IC-341941 & 152 & Local Totru (c) \\
\hline 39 & EC-000377 & 79 & EC-30256 & 113 & IC-341946 & 153 & HPU-51 (c) \\
\hline 40 & IC-100072 & 80 & EC-18257 & 114 & EC-000372 & & \\
\hline
\end{tabular}

Source/Pedigree: NBPGR, Shimla (H.P.) 
Table.2 Reaction of adzuki bean genotypes to Cercospora leaf spot disease under natural conditions

\begin{tabular}{|c|c|c|c|c|}
\hline $\begin{array}{l}\text { Disease } \\
\text { incidence } \\
(\%)\end{array}$ & $\begin{array}{l}\text { Rating } \\
\text { scale }\end{array}$ & Reaction & $\begin{array}{l}\text { No. of } \\
\text { genotypes }\end{array}$ & Genotypes \\
\hline $\mathbf{0}$ & 0 & Highly resistant & - & - \\
\hline $1-5 \%$ & 1 & Resistant & 5 & EC-340286, EC-340267, EC-340271, EC-000263, EC-340251 \\
\hline $6-10 \%$ & 3 & $\begin{array}{l}\text { Moderately } \\
\text { resistant }\end{array}$ & 9 & $\begin{array}{l}\text { EC-000377, EC-340284, IC-341960, EC-57959, IC-485385, EC-340277, IC-341951, IC-341937, IC- } \\
341963\end{array}$ \\
\hline $11-25 \%$ & 5 & $\begin{array}{l}\text { Moderately } \\
\text { susceptible }\end{array}$ & 50 & $\begin{array}{l}\text { EC-000251, EC-340282, IC-16761, Local Totru, HPU-51, IC-46917, EC-340276, EC-34027, EC-240251, } \\
\text { IC-24105, EC-000276, EC-15256, EC-340250, EC-340263, EC-30253, EC-30270, EC-340261, EC-08707, } \\
\text { IC-469173, IC-469174, IC-341944, EC-120480, IC-469172, EC-87896, EC-340246, EC-340287, IC- } \\
\text { 341939, IC-341947, EC-340256, EC-87815, EC-87895, IC-140848, IC-341946, EC-341962, EC-340266, } \\
\text { IC-341959, EC-57459, IC-455396, EC-15257, IC-341940, EC-290251, IC-251353, IC-341953, EC-000248, } \\
\text { IC-108856, EC-000254, EC-340240, EC-57159, EC-340254, EC-000249 }\end{array}$ \\
\hline $26-50 \%$ & 7 & Susceptible & 85 & $\begin{array}{l}\text { EC-187896, IC-341948, EC-340272, EC-340258, EC-15648, EC-281186, EC-120460, IC-341957, EC- } \\
\text { 340275, IC-341950, EC-340245, IC-341949, EC-340257, EC-340265, EC-290652, IC-341958, IC-108854, } \\
\text { EC-340285, EC-340253, IC-100072, EC-24102, EC-341943, EC-340280, EC-59459, EC-340247, EC- } \\
\text { 340279, EC-340259, EC-30250 IC-485886, EC-187898, EC-340268, EC-000262, EC-340249, EC-340283, } \\
\text { EC-340248, EC-87071, EC-340270, IC-469175, EC-340264, EC-340252, EC-340288, EC-108080, EC- } \\
\text { 24523, IC-341945, EC-30256, EC-18257, EC-000271, IC-241041, EC-120466, IC-341956, EC-341955, IC- } \\
\text { 341952, EC-340278, EC-340244, IC-89957, IC-108855, IC-341954, IC-341938, IC-485382, IC-485388, } \\
\text { EC-18256, IC-341941, EC-000372, EC-34264, EC-36070, EC-18959, IC-485396, EC-36973, IC-30270, } \\
\text { EC-340269, IC-341942, IC-0293, IC-140846, IC-34196, IC-140845, EC-34625, EC-18151, IC-24522, EC- } \\
\text { 340260, EC-340262, EC-340273, EC-340255, IC-108857, EC-80850, EC-000264 }\end{array}$ \\
\hline$>50 \%$ & 9 & $\begin{array}{l}\text { Highly } \\
\text { susceptible }\end{array}$ & 4 & EC-340274, EC-59489, EC-340281, EC-240246 \\
\hline
\end{tabular}


Table.3 Clustering pattern of 153 adzuki bean genotypes according to Mahalanobis D2- analysis

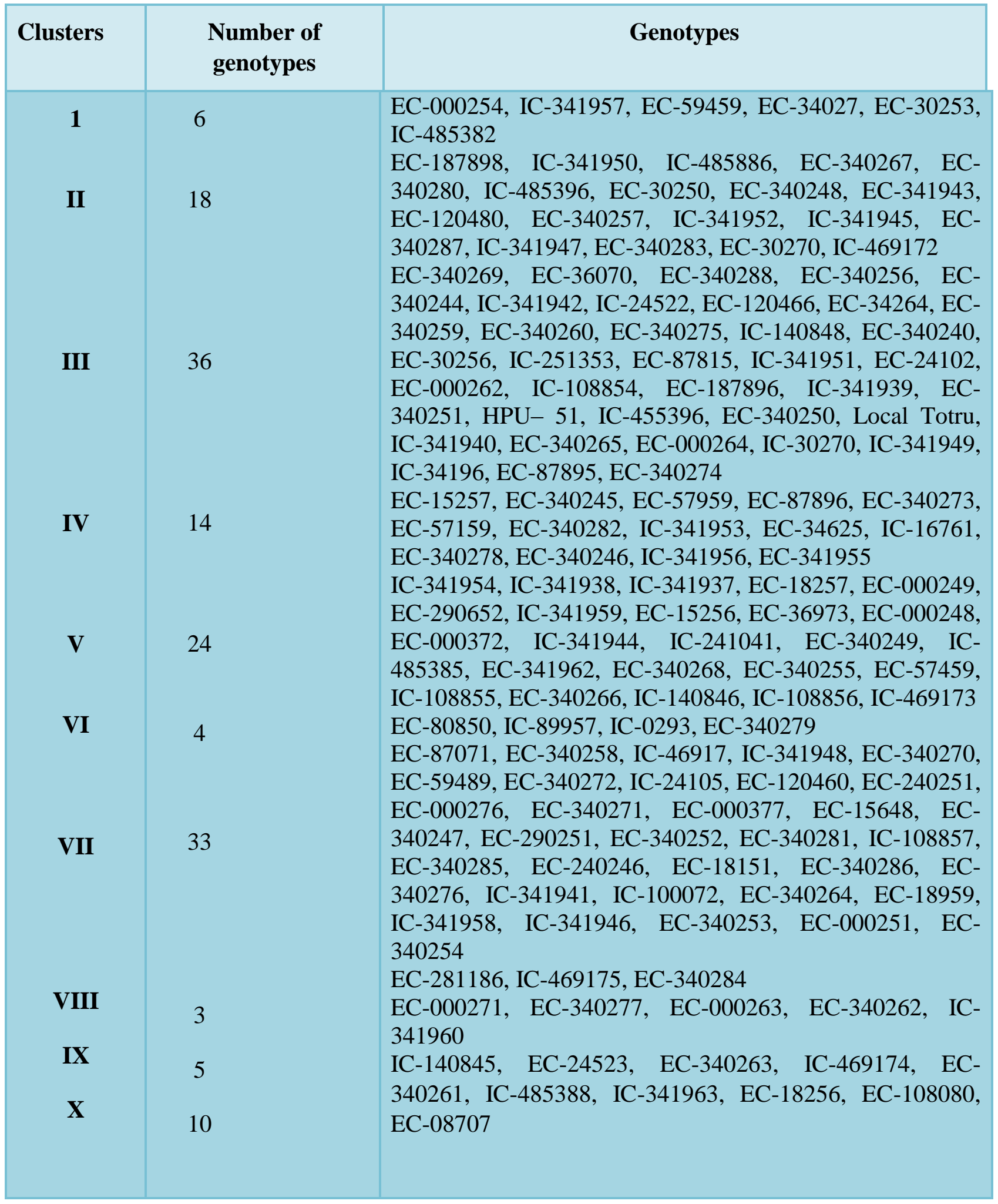


Int.J.Curr.Microbiol.App.Sci (2020) 9(10): 603-617

Table.4 Average intra and inter cluster distances

\begin{tabular}{|c|c|c|c|c|c|c|c|c|c|c|}
\hline & $\begin{array}{c}\text { Cluster } \\
1 \\
31.72\end{array}$ & $\begin{array}{c}\text { Cluster } \\
2 \\
24.91\end{array}$ & $\begin{array}{c}\text { Cluster } \\
3 \\
30.1\end{array}$ & $\begin{array}{l}\text { Cluster } \\
4 \\
29.25\end{array}$ & $\begin{array}{l}\text { Cluster } \\
5 \\
25.65\end{array}$ & $\begin{array}{c}\text { Cluster } \\
6 \\
25.87\end{array}$ & $\begin{array}{c}\text { Cluster } \\
7 \\
27.14\end{array}$ & $\begin{array}{c}\text { Cluster } \\
8 \\
25.13\end{array}$ & $\begin{array}{c}\text { Cluster } \\
9 \\
\mathbf{3 3 . 3 4}\end{array}$ & $\begin{array}{c}\text { Cluster } \\
10 \\
28.66\end{array}$ \\
\hline Cluster 1 & 0.00 & 55.45 & 45.28 & 88.75 & 40.05 & 56.28 & 52.25 & 52.16 & 36.42 & 41.07 \\
\hline Cluster 2 & & 0.00 & 66.29 & 107.34 & 40.24 & 70.96 & 66.92 & 62.03 & 60.53 & 42.29 \\
\hline Cluster 3 & & & 0.00 & 74.03 & 44.69 & 45.90 & 34.21 & 46.05 & 40.10 & 60.11 \\
\hline Cluster 4 & & & & 0.00 & 88.73 & 54.73 & 72.47 & 91.60 & 73.60 & 103.75 \\
\hline Cluster 5 & & & & & 0.00 & 56.28 & 46.53 & 49.62 & 44.91 & 41.22 \\
\hline Cluster 6 & & & & & & 0.00 & 40.73 & 66.91 & 43.30 & 72.85 \\
\hline Cluster 7 & & & & & & & 0.00 & 55.68 & 47.44 & 62.72 \\
\hline Cluster 8 & & & & & & & & 0.00 & 57.79 & 47.80 \\
\hline Cluster 9 & & & & & & & & & 0.00 & 53.26 \\
\hline Cluster 10 & & & & & & & & & & 0.00 \\
\hline
\end{tabular}


Table.5 Mean values of ten clusters for different traits of adzukibean genotypes

\begin{tabular}{|c|c|c|c|c|c|c|c|c|c|c|}
\hline & $\begin{array}{l}\text { Days to } 50 \% \\
\text { flowering }\end{array}$ & $\begin{array}{l}\text { Days to } 80 \% \\
\text { maturity }\end{array}$ & $\begin{array}{l}\text { Plant } \\
\text { height }\end{array}$ & $\begin{array}{l}\text { Primary } \\
\text { branches/ } \\
\text { plant }\end{array}$ & $\begin{array}{l}\text { Clusters/ } \\
\text { plant }\end{array}$ & Pods/cluster & Pods/plant & Seeds/pod & $\begin{array}{l}\text { 1000-seed } \\
\text { weight }\end{array}$ & $\begin{array}{l}\text { Seed } \\
\text { yield/plant }\end{array}$ \\
\hline Cluster 1 & 69.77 & 108.72 & 88.72 & 3.87 & 14 & 2.79 & $27.94^{*}$ & 7.54 & 83.93 & 12.17 \\
\hline Cluster 2 & $64.66 *$ & 106.83 & $95.27 * *$ & 3.39 & 15.64 & 3.22 & 39.59 & 7.69 & $67.94 *$ & $12.94 * *$ \\
\hline Cluster 3 & 65.54 & 106.47 & 76.65 & 3.51 & 16.30 & 3.03 & 37.65 & 7.25 & 99.13 & 12.11 \\
\hline Cluster 4 & 69.52 & 113.17 & 84.14 & 3.68 & $13.69 *$ & 3.17 & 34.97 & 7.70 & $146.15 * *$ & $12.94 * *$ \\
\hline Cluster 5 & 64.85 & 107.63 & 85.10 & 3.36 & 17.55 & 2.86 & 39.39 & 7.53 & 83.56 & 12.74 \\
\hline Cluster 6 & 66.74 & 107.61 & 89.16 & $2.87 *$ & 17.82 & $3.41 * *$ & 39.38 & 7.46 & 118.12 & 12.35 \\
\hline Cluster 7 & 65.41 & $104.31 *$ & 83.67 & 3.39 & $18.99 * *$ & 3.31 & $50.64 * *$ & $6.91 *$ & 106.27 & 11.35 \\
\hline Cluster 8 & 66.27 & 106.17 & $62.47 *$ & 3.40 & 13.74 & $2.15^{*}$ & 33.76 & 7.36 & 83.19 & $10.95 *$ \\
\hline Cluster 9 & 69.34 & 110.80 & 87.67 & 3.60 & 14.88 & 2.94 & 33.24 & 7.78 & 96.39 & 12.08 \\
\hline Cluster 10 & $72.58 * *$ & $116.60 * *$ & 83.10 & $4.42 * *$ & 13.85 & 2.57 & 31.93 & $8.10 * *$ & 71.18 & 12.72 \\
\hline
\end{tabular}

**and *: maximum and minimum values respectively 
Int.J.Curr.Microbiol.App.Sci (2020) 9(10): 603-617

Fig.1 Frequency distribution of adzukibean germplasm for qualitative traits

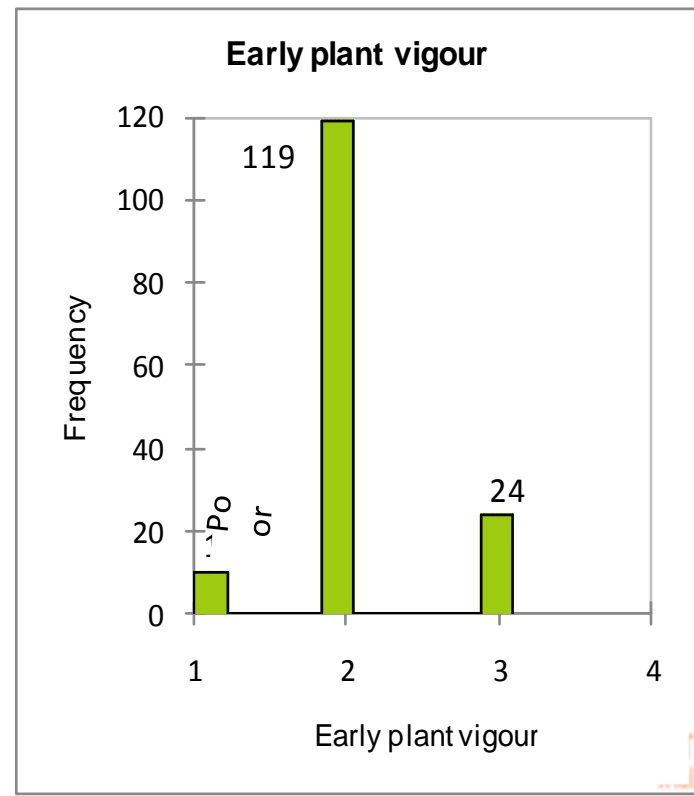

Fig.1(a)

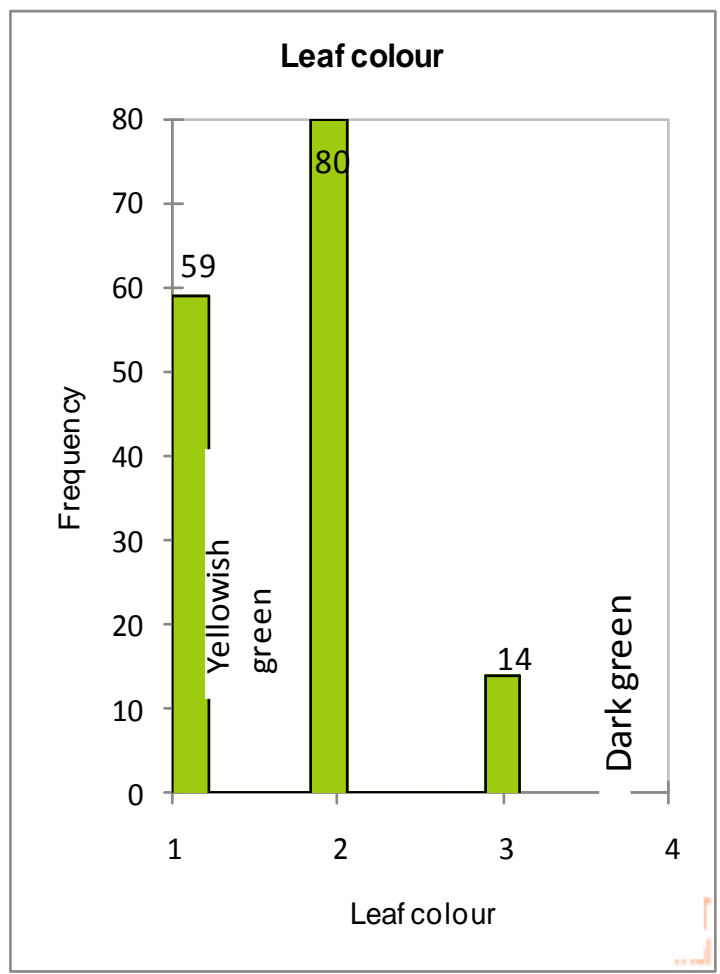

Fig.1(c)

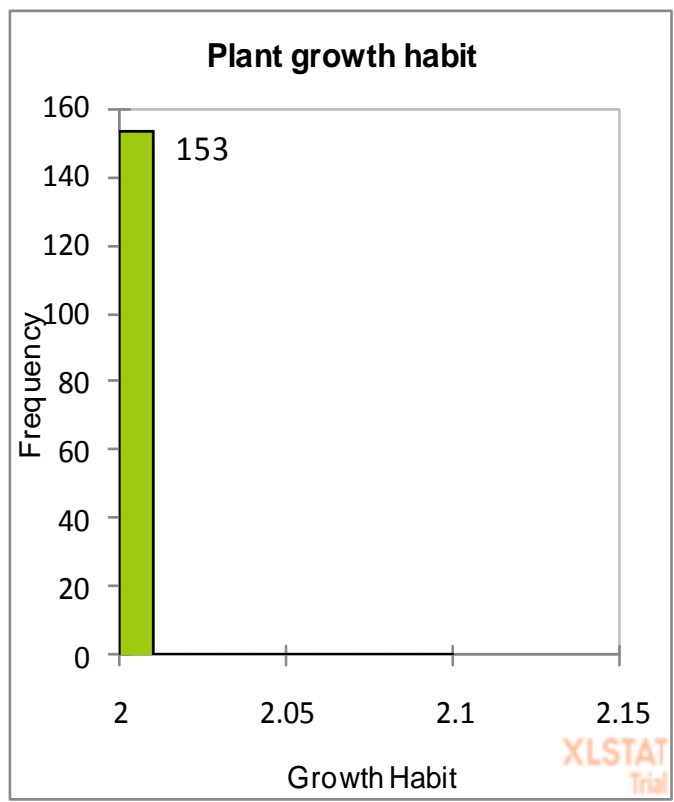

Fig.1(b)

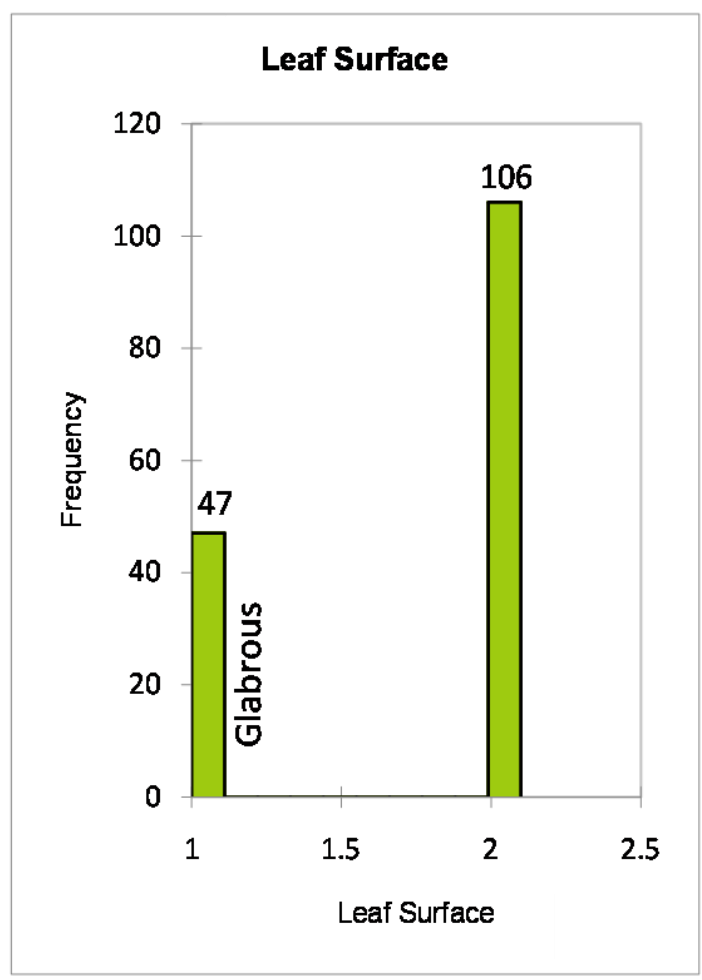

Fig.1(d) 
Int.J.Curr.Microbiol.App.Sci (2020) 9(10): 603-617

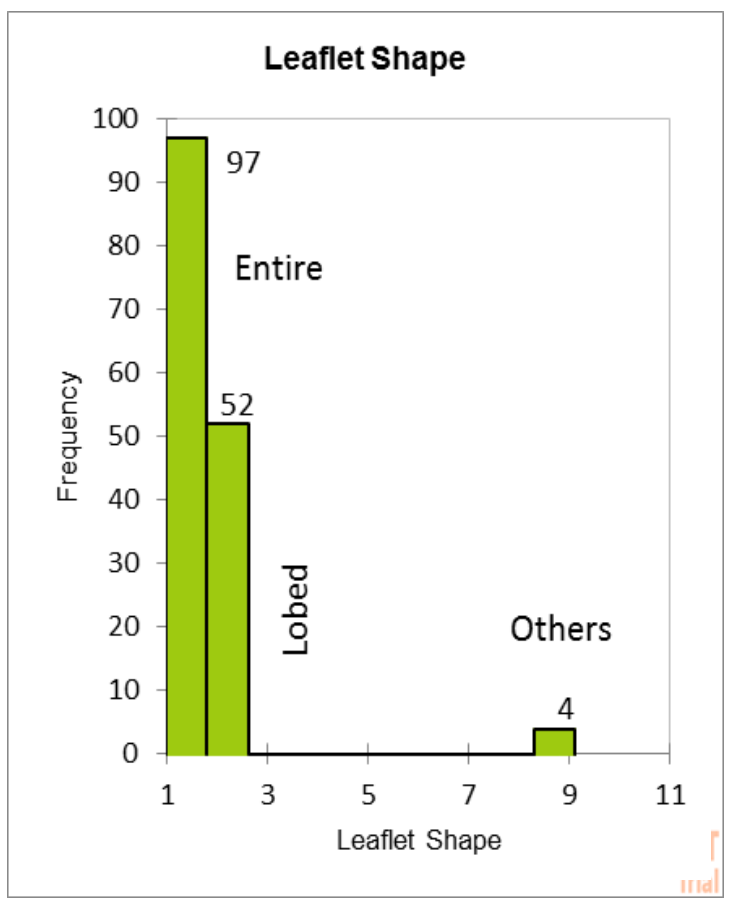

Fig.1(e)

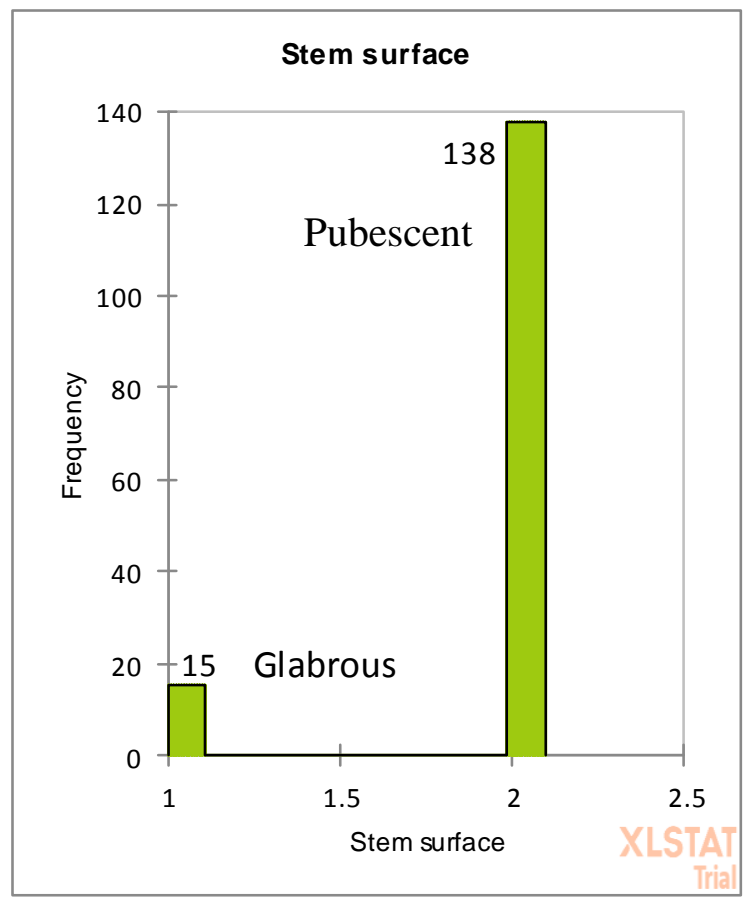

Fig.1(g)

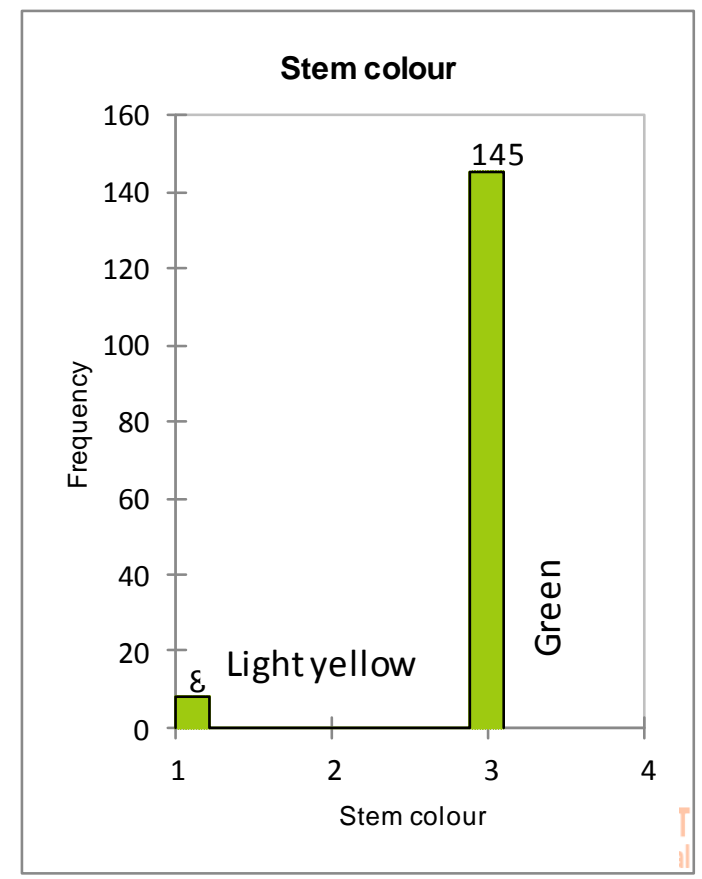

Fig.1(f)

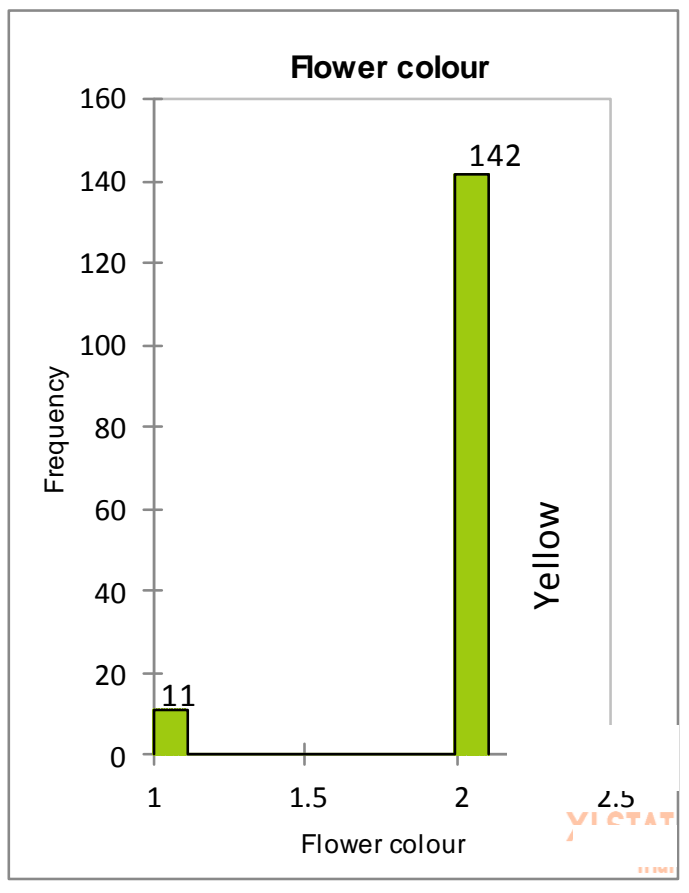

Fig.1(h) 
Int.J.Curr.Microbiol.App.Sci (2020) 9(10): 603-617

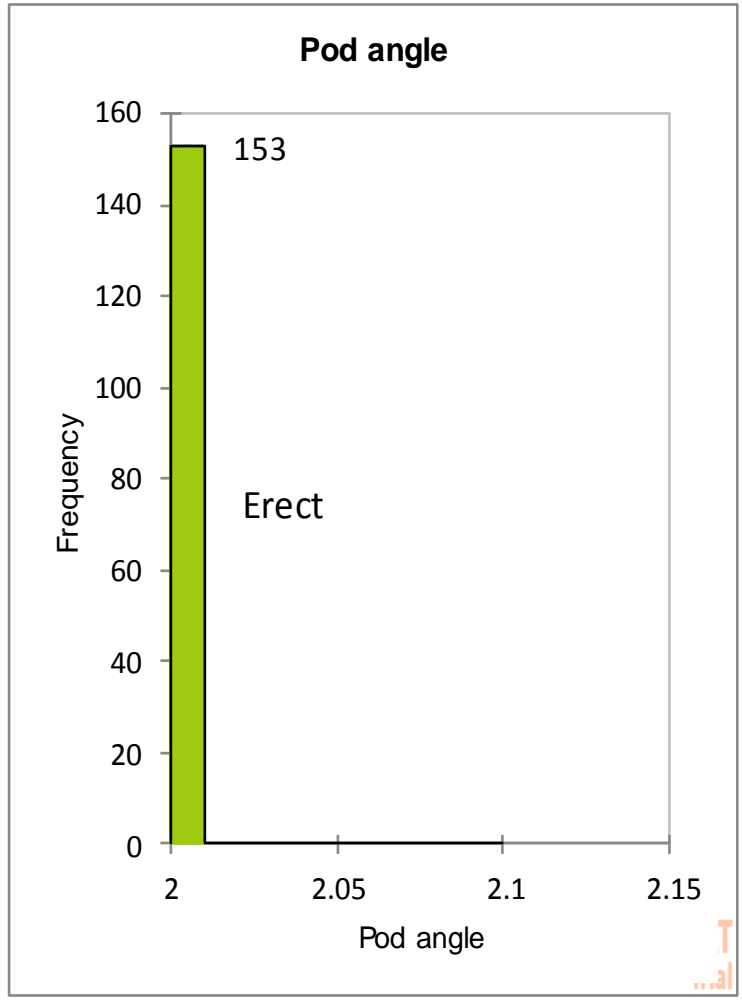

Fig.1(i)

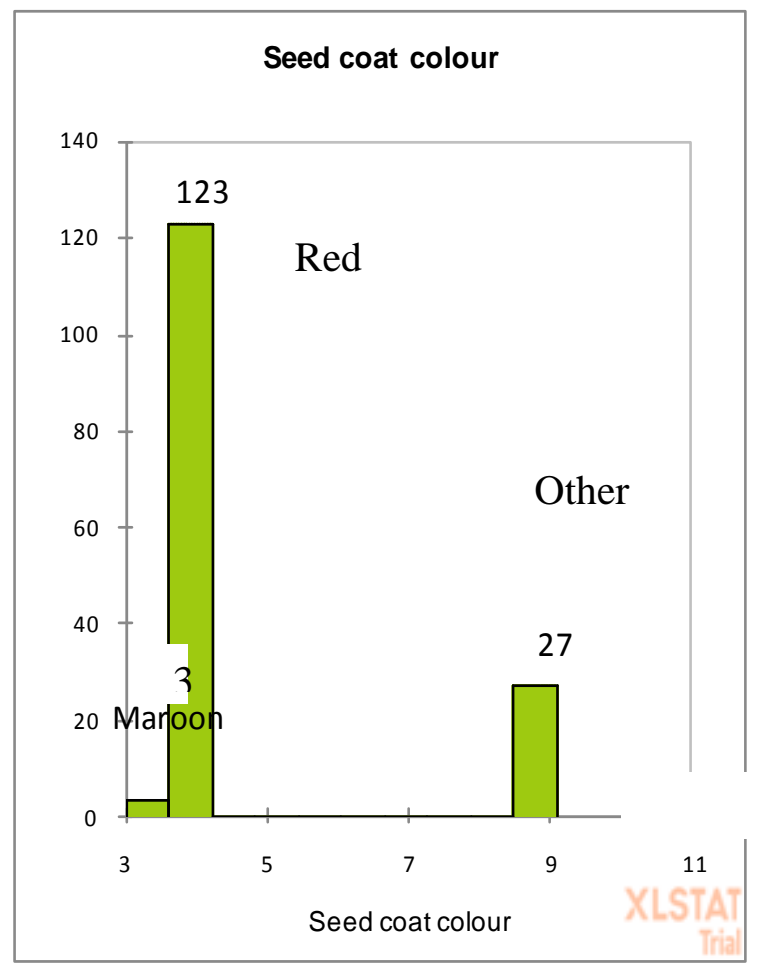

Fig.1(k)

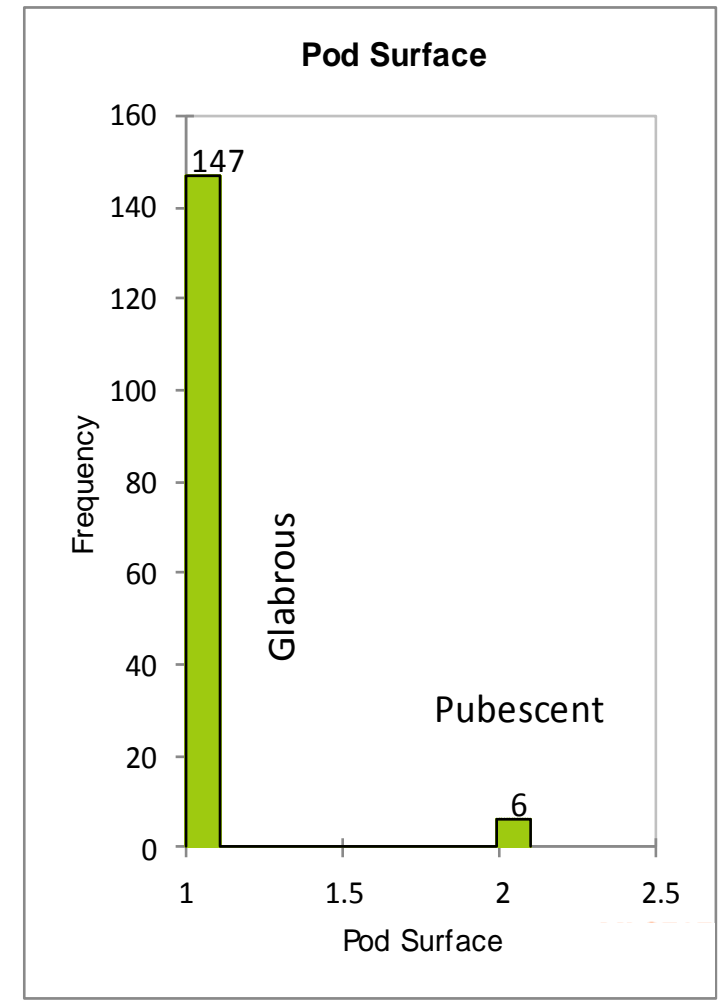

Fig.1(j)

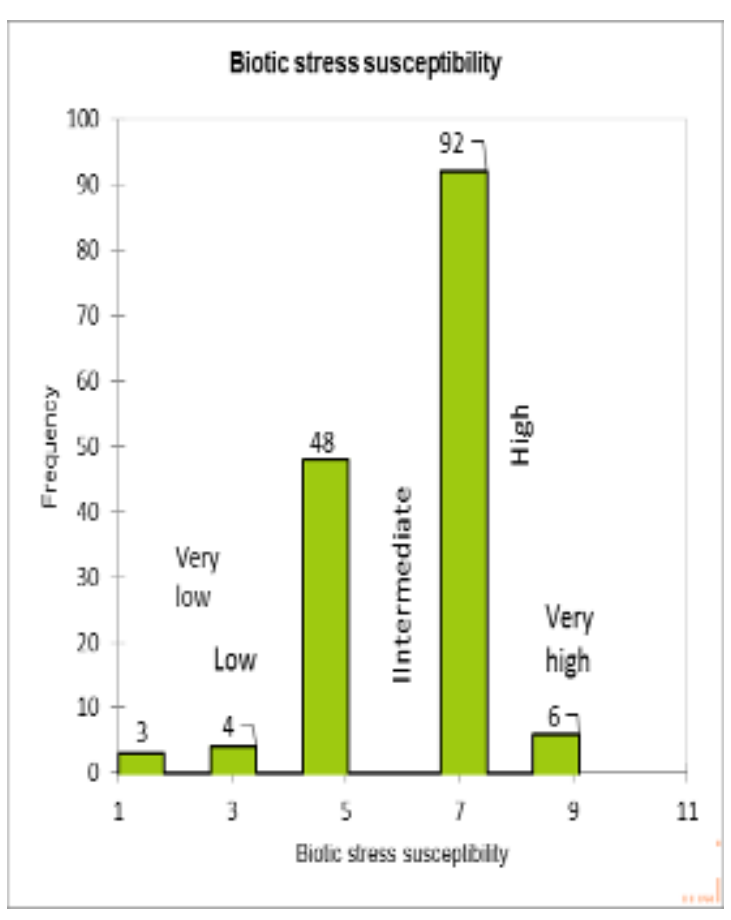

Fig.1(l) 
Fig.2 Dendrogram depicting relationships among the adzuki bean genotypes

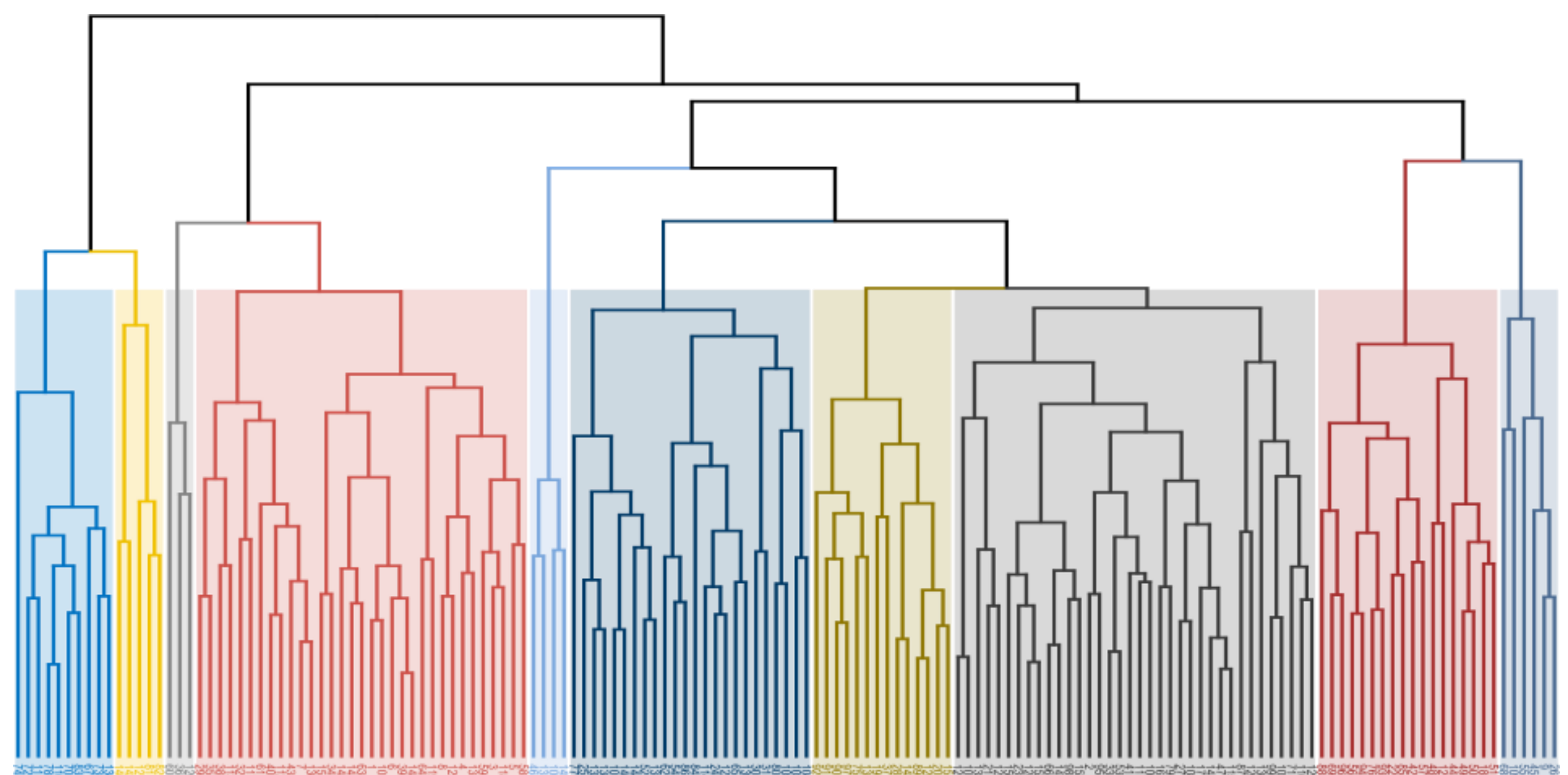




\section{Cluster analysis}

Non-hierarchical Euclidean cluster analysis grouped 153 genotypes of adzuki bean including checks into ten clusters with cluster III having highest number of genotypes i.e. 36 , followed by cluster VII with 33 genotypes and cluster $\mathrm{V}$ with 24 genotypes (Table 3 and Fig. 1). Average intra and inter cluster distances are presented in Table 4. Different clustering patterns were also reported in adzuki bean by some earlier workers (Zhenxing, 2011; Redden et al., 2009; Wang et al., 2019).

Maximum intra cluster distance was observed in cluster IX (33.34) followed by cluster I (31.72) indicating genetic diversity among the genotypes belonging to these clusters. Therefore, the crosses should be made between the genotypes of these clusters for developing good segregants. The maximum inter cluster distance was observed between cluster II and cluster IV (107.34) followed by distance between cluster IV and cluster $1 \mathrm{X}$ (103.75) suggesting wide diversity between these clusters. Therefore, genotypes belonging to these clusters may be used in hybridization programme for the improvement of adzuki bean. The least intercluster distance was observed between cluster III and VII (34.21) indicating close relationship between the genotypes of these clusters.

The diversity was also supported by the considerable amount of variation among the cluster mean for different characters. The cluster means of adzuki bean genotypes falling under different clusters are presented in Table 5. Cluster 2 showed the maximum cluster mean values for plant height and seed yield per plant. For 1000-seed weight and seed yield per plant, cluster 4 recorded the maximum values. Cluster 6 showed the maximum values for pods per cluster.
Maximum values for pods per plant and clusters per plant were recorded by cluster 7 . Cluster 10 showed the maximum cluster mean values for days to $50 \%$ flowering, days to $80 \%$ maturity, primary branches per plant and number of seeds per pod. Thus, these genotypes hold great promise as parents for procuring elite lines through hybridization and to create further variability for these characters.

Diversity analysis is an essential process for identification of the genetic relatedness of the available genetic resources which is helpful in better utilization and conservation of germplasm. Frequency distribution graphs of various qualitative traits revealed sufficient variation among adzuki bean accessions and provided useful information about the diversity of these accessions. Reaction of adzuki bean to other diseases like brown stem rot, charcoal rot Fusarium wilt etc. was studied in China by many scientists but studies related to disease incidence and reaction of adzuki bean to Cercospora leaf spot is not much done. Here in this study, response to Cercospora leaf spot disease showed that 5 genotypes viz., EC-340286, EC-340267, EC-340271, EC-000263 and EC340251 were resistant to the disease.

In the present study, sufficient variability was observed in adzuki bean accessions. Cluster analysis clearly showed that some genotype is closely related while some as significantly distinct. 153 adzuki bean genotypes including checks were grouped into ten clusters with cluster III having highest number of genotypes i.e. 36, followed by cluster VII and cluster V. Maximum intra cluster distance was observed in cluster IX (33.34). The maximum inter cluster distance was observed between cluster II and cluster IV (107.34) indicating genetic diversity among the genotypes belonging to these clusters. Therefore, crosses should be made between 
these genotypes for developing good segregants.

\section{Acknowledgement}

I (Anjali) would like to express my sincere gratitude and appreciation to my major advisor Dr. Neelam Bhardwaj for being a prodigious mentor for me.

\section{References}

Anonymous. 2019. Food And Agricultural organization of the United Nations. https://www. FAOstat. Fao.org.

Alice, D., and Nadarajan, N. 2007. Pulses: Screening techniques and assessment for disease resistance. All India Coordinated Research Project on MULLaRP-Tamil Nadu Agricultural University. Kasturi Graphics and Printers, Coimbatore.

Barrett, B.A., and Kidwell, K.K. 1998. AFLPbased genetic diversity assessment among wheat cultivars from the Pacific Northwest. Crop Science. 38(5): 12611271.

Dua, R.P., Raiger, H.L., Phogat, B.S., Sharma, S.K. 2009. Underutilized Crops: Improved Varieties and Cultivation Practices. ICAR publication, p. 66.

Federer, W.T. 1956. Augmented design. Hawaiin Planters Records.40: 191-207.

Franco, T., and Hidalgo, R. 2003. Análisis estadístico de datos de caracterización morfológica de Recursos Fitogenéticos. Cali, Colombia: Instituto Internacional deRecursosFitogenéticos (IPGRI), p 89.

Gohara, A.K., Pereira de Souza, A.H., Gomes, S.T.M., de Souza, N.E., Visentainer, J.V., and Matsushita, M. 2016. Nutritional and bioactive compounds of adzuki beans cultivars using chemometric approach. Food Science and Technology. 40(1):1981-
1829.

Kaushal RP and Singh BM. Interspecific hybridization between urdbean (Vigna mungo (L.) Hepper) and adzuki bean (Vigna angularis (Willd.) Ohwi and Ohashi). Indian Journal of Agricultural Science, 1988; 58:123-124.

Kumari, S., Nirala, R.B., Rani, N., and Prasad, B.D. 2017. Selection criteria of linseed (Linum usitatissimum L.) genotypes for seed yield traits through correlation and path coefficient analysis. Journal of Oilseeds Research. 34(3):171-174.

Mahalanobis, P.C. 1936. On the generalized distance in statistics. Proceedings of the National Institute of Science of India. 2(1): 49-55.

Redden, R.J., Basford, K.E., Kroonenberg, P.M., Islam, F.M.A., Ellis, R., Wang, S., Cao, Y., Zong, X., and Wang, X. 2009. Variation in adzuki bean (Vigna angularis) germplasm grown in China. Crop Science. 49(3):771-782.

Shweta. 2013 Study on the Biochemical profile of different Vigna spp. prevalent under mid-hill conditions of Himachal Pradesh. PhD Thesis, Department of Chemistry and Biochemistry, CSK Himachal Pradesh Krishi Vishwavidyalaya, Palampur, India.

Thompson, J.A., Nelson, R.L., and Vodkin, L.O. 1998. Identification of diverse soybean germplasm using RAPD markers. Crop Science. 38(5):13481355.

Vaughan, D.A., Tomooka, N., Kaga, A., Singh, R.J., and Jauhar, P.P. 2004. Adzuki bean. Genetic resources, chromosome engineering and crop improvement: grain legumes. CRC Press, Boca Raton, FL, pp. 341-353.

Wang, S.M., Redden, R.J., Hu Jiapeng, J.P., Desborough, P.J., Lawrence, P.L., and Usher, T. 2001. Chinese adzuki bean germplasm: 1. Evaluation of agronomic 
traits. Australian Journal of Agricultural Research. 52(6): 671-681.

Wang, L., Wang, J., and Cheng, X. 2019. Adzuki bean (Vigna angularis (Willd.) Ohwi and Ohashi) breeding. Advances in Plant Breeding Strategies: Legumes. pp 1-23.

Yamaguchi, H. 1992. Wild and weedy adzuki beans in Japan. Economic Botany. 46(4): 384-394.

Zhen-xing, L., Gui-mei, Z., and Jian, C. 2011. The morphological diversity of adzuki bean landraces from Tangshan. Journal of Hebei Agricultural University. 34(4): $1-4$.

\section{How to cite this article:}

Anjali and Neelam Bhardwaj. 2020. Morphological Characterization and Diversity Analysis of Adzukibean [Vigna angularis (Willd.) Ohwi and Ohashi] Germplasm. Int.J.Curr.Microbiol.App.Sci. 9(10): 603-617. doi: https://doi.org/10.20546/ijcmas.2020.910.072 